Session 3266

\title{
A TOOL FOR TEACHING STRESS TRANSFORMATION BY MOHR'S CIRCLE
}

\author{
James Moller, Amer Mokaddem \\ Miami University, Oxford, $\mathrm{OH}$
}

\begin{abstract}
The determination of transformed and principal stresses is frequently a significant conceptual hurdle for Mechanics of Materials students. Mohr's circle is commonly introduced as a tool ostensibly to make the transformation concept easier to understand and apply. Yet learning the Mohr's circle conventions frequently introduces an additional challenge. A computer program to assist the learning of stress transformation and Mohr's circle is introduced. It displays diagrams of the state of stress and the corresponding Mohr's circle as transformation angle is varied. Results of student testing indicate it is most effective at improving ability to anticipate the variation of stress magnitude as transformation angle varies and for identifying principal stresses on the circle.
\end{abstract}

\section{Introduction}

The transformation of stress, strain, moment of inertia among coordinate systems is important in static and structural analysis. Late in the last century, Mohr ${ }^{1,2}$ introduced a graphical construction to assist in the process. At Mohr's time, the technology for graphical construction was drafting and any technology for computation was quite tedious compared with modern tools. His graphical approach to constructing his circle could be used to find approximate values for transformed stresses and thereby save what, at the time, would have been great computational effort. Indeed, as recently as 1972, the construction of Mohr's circle was couched in drafting terminology ${ }^{3}$.

Mohr's circle is among the most difficult topics for students to comprehend, according to surveys of them and examination of their test results. Students in the Manufacturing Engineering department's Strength of Materials course were surveyed at the end of both the Fall 1995 and Spring 1996 semesters. Among the questions posed to them was their level of confidence with each of the principal topics covered. In both semesters, stress transformation and Mohr's circle ranked among the lowest. This was in spite of having spent two lectures on them each semester.

Based on student questions and comparison with the other topics covered in the course, there are several distinguishing features of the stress transformation concepts which make it more challenging to learn. First, the relation among the load on a member and the state of stress at a point for a single coordinate system takes some students the entire semester to master. Second, the parametric relation and stress axes become confused with the spatial coordinate axes. Third, 
comprehension is contingent upon the student being able to see some imaginary "rotation" of an infinitesimal cube while the member does not rotate. Fourth, the potential application of values gleaned from Mohr's circle is not apparent until after several subsequent class sessions. Fifth, it's not clear at first learning why one would want what is fundamentally the same information in a different coordinate system.

It is easy for the students to lose sight of the purpose of the circle; the determination of principal stresses and angles and the state of stress for a given transformation. It is possible, in the present educational and professional environment, to entirely omit Mohr's circle from a course and rely on the students to program the transformation equations. It is also entirely conceivable that Mohr's circle could be taught as the principal or only means for stress transformation. Perhaps there is a balance which could be struck between these two extremes which is particularly beneficial to the student. It is important to keep in mind how the learning styles of students have changed over the past decade as the personal computer has become common in all facets of daily life and as user interfaces have become increasingly image-based. Students are very comfortable with associating objects on the screen at disparate locations with an implicit process by which they are associated (e.g., an equation). They are also comfortable with associating entities other than words (e.g., colors, symbols, font style) with information.

There are opportunities, then, to take advantage of these learning styles in the teaching of engineering concepts. Many of these are afforded by the highly object-oriented and graphic computer interface environments and the ubiquity of programming languages which support such environments. Ressler ${ }^{4}$, Smith ${ }^{5}$, and Rossow ${ }^{6}$ have introduced programs to foster visualization of Mohr's circle for plane stress with graphic screen displays. A program which creates a more interactive environment as well as the results of an experiment in its effectiveness are introduced here. A discussion of the strategy for using it in teaching is also included.

\section{Program Interface}

The learner enters the state of stress in the original coordinate system. The transformation angle can be entered either at a slidebar or in a field box. As the operator varies the angle, the cube reorients itself by the specified amount. As the cube rotates the shear and normal stress vectors increase or decrease in length proportional to the transformed stress. When the cube is rotated to a principal angle, the color of the cube border changes. A second output window shows the corresponding Mohr's circle with two rays running from the center to the points representing the state of stress at the transformation angle. To make it easier to match the point on the circle with the cube face, the rays are colored to match the normal stress vector colors. A third output window shows numerical values of principal and transformed stresses and principal angles. A fourth output window gives the coordinates of a mouse-driven cursor lying in the Mohr's circle window. Both cube, Mohr's circle, and numerical output boxes can be toggled on and off. An example of the screen seen by the learner is shown in Figure 1. 


\section{Teaching Experiment}

An experiment was conducted to check the efficacy of several specific program features. They were: 1) that the use of color would help to match information between the cube diagram and Mohr's circle, 2) that simultaneous animation of cube and circle would improve learning of the relations among transformation angle, state of stress, and the angles on the circle, 3) that cube animation would improve learning of principal stress and maximum shear stress locations.

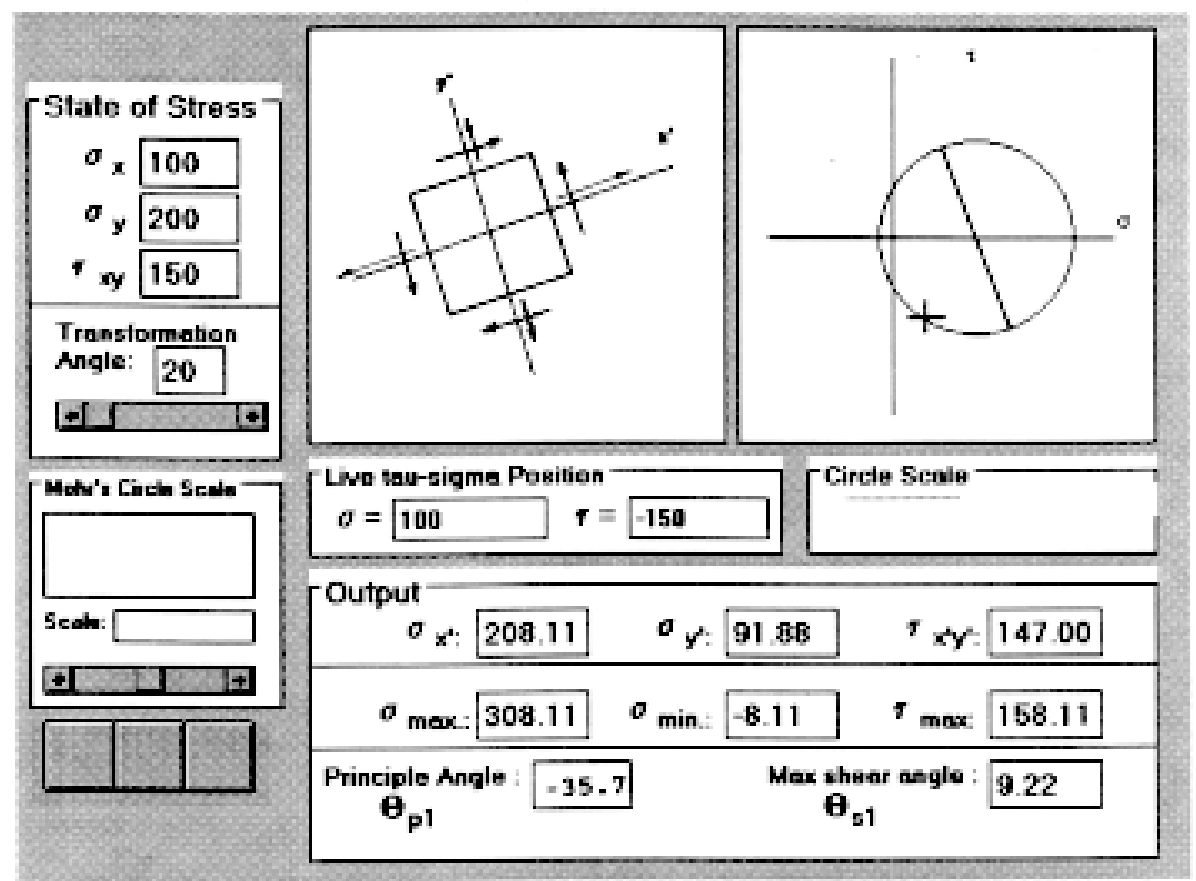

Figure 1. Example of the state of stress windows during program operation. Infinitesimal cube, Mohr's circle, and numerical output windows are shown in their unhidden state.

The program effectiveness was assessed during a scheduled class meeting. There were fourteen students. Most were junior level. The students had just completed a lecture and one homework assignment on principal stresses and Mohr's circle. Before seeing the program in operation, they were asked a set of written questions as a baseline from which to measure their progress. Questions included asking for Mohr's circle for specific states of stress as well as points on the circle for principal stresses and maximum shear stress.

After a brief overview, the instructor ran the program and discussed its operation in terms of the items displayed and their relation. The monitor display was projected to an overhead screen. Initially, only the input panel and infinitesimal cube were shown. The purpose of keeping the circle display off initially was that prior teaching experience has given evidence that, when students are presented with the circle simultaneously with the concept of stress transformation, they focus entirely on trying to understand the circle forget its principal purpose. Students were asked to note the stress vectors' directions and magnitudes while the cube was rotated through transformation angles. The Mohr's circle window was then enabled and the demonstration was repeated with their attention called to the relation of points on the circle to stress vectors lengths. 
At the demonstration midpoint, learners were quizzed again and asked to identify directions of stress vectors for given points on the circle, how stress magnitudes would vary as the transformation angle was varied, and the locations on the circle at which shear stress was zero. The demonstration then continued with a discussion of principal stresses and planes. The numerical output box was turned on and attention was called to the facts that 1) when the transformation angle is zero, the corresponding points on the circle are not necessarily at the 3 and 9 o'clock locations, 2) principal angles are perpendicular to maximum shear stress angle, and 3) shear stress on the cube is zero at the principal angles. At the classes' end, students were asked the same questions as in the pre-test.

\section{Results}

The answers to the identical pre- and post-test questions were compared to identify areas of increased understanding. Students were found to be distinctly more capable of creating the sketches for uniaxial tension and pure shear. In some questions, such as ones asking for the purpose of the circle and the locations of principal stresses and maximum shear stress, no distinct improvement was seen. Three students appeared to have crossed the conceptual hurdle; they showed improvement in several questions. The principal result of the intermediate questions was that while students could not assign the correct stress vector direction, they did very well at identifying how stress magnitude varied. Over half the class correctly identified the trends for normal stresses and shear stress with varying transformation angle and as well as the circle locations corresponding with the state of stress when shear stress is zero.

\section{Conclusions}

That this program is an effective tool for conveying certain aspects of stress transformation and the Mohr's circle graphic tool has been indicated by the first trial results. While they indicate program use improved students' ability to state the relations among stresses as transformation angle varied, it didn't build their ability to associate names with the circle landmarks (e.g., principal stresses). The program's effectiveness to improve student learning outside of class has yet to be determined. We are concerned that making it available too early may hinder learning. If the homework is focused on exercises in which the correct numerical values is taken by the student as being important, it could be easily abused as a black box which cuts down struggles with homework.

The results showed that the program needs to be enhanced for students to have an understanding of the purpose of Mohr's circle. The program presently has no feature which calls upon the learner's physical intuition to link the loading of a member with the state of stress at a point. A planned enhancement will be to use a manually loaded member ( rather than a mouse input ) to generate the stress. A schematic is shown in Figure 2. The loaded device is a thin-walled tube having a strain gage rosette mounted on the surface. The tube ends have hand grips and braces to ride against the lower arms. With the braces, bending moments parallel and perpendicular to the tube long axis can be more easily applied. The learner holds the member and manually loads it 
while watching the infinitesimal cube and Mohr's circle on the monitor. Principal stresses are calculated from the rosette strains and a plane stress assumption.

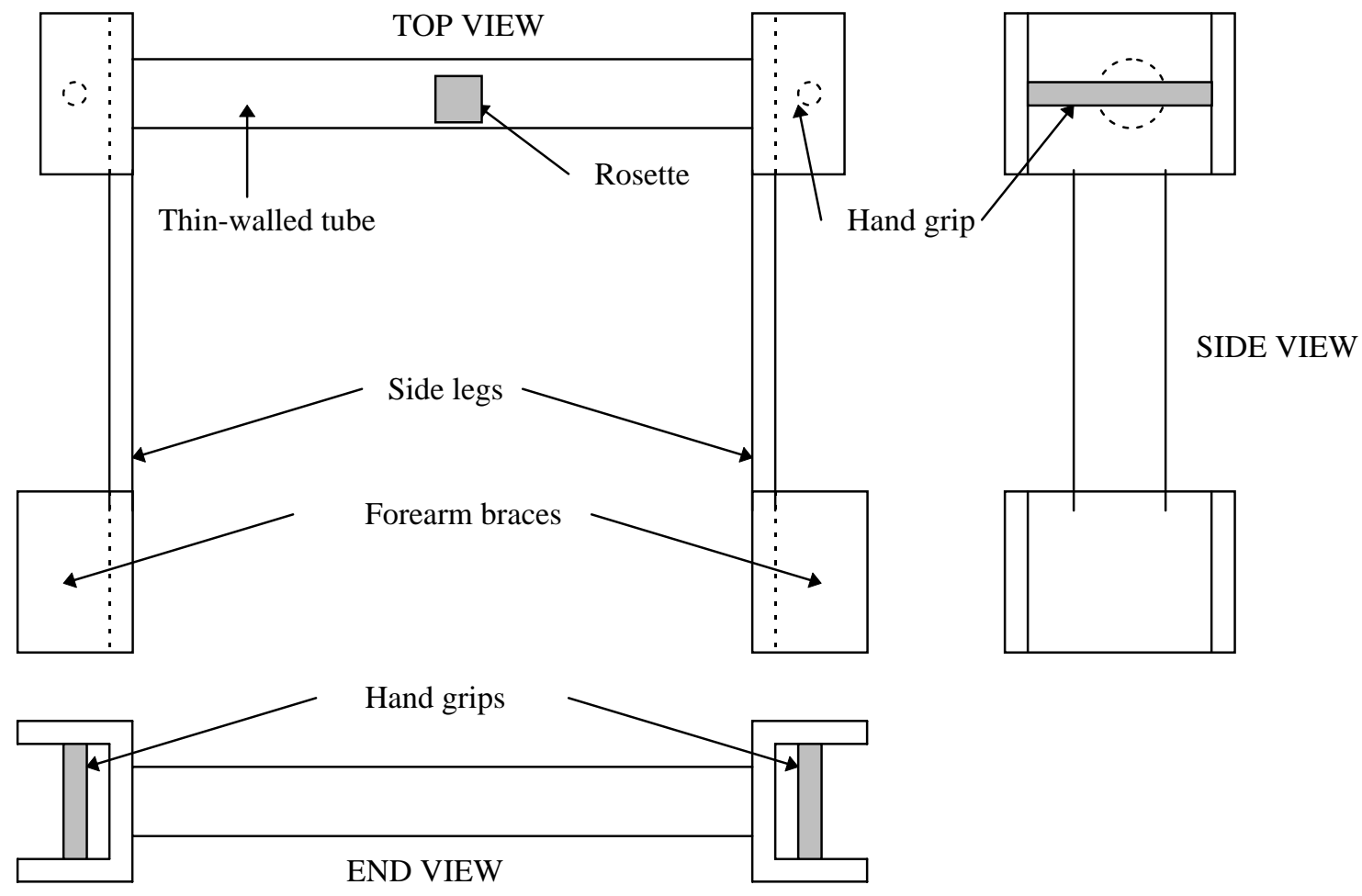

Figure 2. Schematic diagram showing the structural member to be manually loaded by the learner. The learner presses the forearm braces toward each other to create bending and attempts to rotate the side legs with respect to each other to create torsion.

\section{Acknowledgments}

The authors would like to thank Professor Bruce Bardes for his assistance with the experiments.

\section{References}

1. O. Mohr, "Uber die Darstellung des Spannungszustandes und des Deformationszustandes eines Korperelementes," Zivilingenieur, 1882.

2. O. Mohr, Abhandlungen aus dem Gebiete der technischen Mechanik, Wilhelm Ernst and Son, Berlin, 1906.

3. S.P. Timoshenko and J.M. Gere, Mechanics of Materials, Van Nostrand, 1972, p. 53.

4. S. J. Ressler, "The Visual Stress Transformer - An Animated Computer Graphics Program for Engineering Mechanics Education," Session 1668, ASEE Annual Conference Proceedings 1996

5. Smith, S. A. Jr., "Microcomputer-Aided Teaching Of Mohr's Circle," International Journal of Applied Engineering Education. v 2 n 2, 1986, p. 91-101.

6. Rossow, M.P., "An Interactive Program for Teaching Stress Transformation with Mohr's Circle," Computers in Education Journal, v. 6 n. 4, 1996, p. 42-46.

James C. Moller received M.S. \& M.Eng. degrees from M.I.T and a Ph.D. from R.P.I. He is presently assistant professor in the Manufacturing Engineering Department at Miami University.

Amer Mokaddem is presently a Manufacturing Engineering student at Miami University. 